\title{
Signs and symptoms of foot and ankle dysfunction in children with joint hypermobility
}

\author{
Leslie L Nicholson ${ }^{1,3^{*}}$, Verity Pacey ${ }^{2,3}$, Louise Tofts ${ }^{3,4}$, Craig Munns ${ }^{4,5}$, Roger Adams ${ }^{6}$ \\ From 4th Congress of the International Foot and Ankle Biomechanics (i-FAB) Community \\ Busan, Korea. 8-11 April 2014
}

\section{Background}

Foot and ankle complaints are common in people with hypermobility. Children with hypermobility (Beighton score $\geq 4 / 9$ ) were recruited for a longitudinal study from The Children's Hospital at Westmead in Sydney, Australia. Baseline data included Beighton score, BMI for age, Star Excursion Balance Test (SEBT), Foot Posture Index (FPI), anterior drawer, subtalar inversion stress test, Lower Limb Assessment Scale (LLAS), physical activity and child-rated quality of life (PedsQL).

\section{Results}

53 girls and 47 boys (mean age 11.5 $\pm 3.1 \mathrm{yrs}$ ) with a mean Beighton score of $6.7 / 9$ and LLAS of $8.2 / 12$ were recruited. Of these 100 children, 94 met the Brighton criteria for Joint Hypermobility Syndrome [1] and 90 met the Villefranche criteria for Ehlers-Danlos SyndromeHypermobility Type [2]. Of the entire cohort, $50 \%$ reported experiencing ankle joint pain and $13 \%$ foot pain that had lasted 3 or more months, 36\% reported recurrent "rolling" one or both ankles while only $8 \%$ reported foot instability. The average FPI in this cohort was +6.6, with $86 \%$ of the children having FPI scores of 5 or more and $19 \%$ with scores of 10 or more. Paired samples ttests revealed that those children who reported chronic ankle pain were the ones experiencing recurrent episodes of instability $(\mathrm{p}=0.016)$. Recurrent instability did not significantly correlate with anterior talofibular ligament laxity as assessed with the ankle anterior drawer test or the subtalar inversion test or with foot posture (all $\mathrm{p}>0.5$ ). While the Beighton score moderately correlated [3] $(\mathrm{r}=0.31, \mathrm{p}=0.002)$ with the LLAS, only the LLAS correlated with physical activity $(\mathrm{r}=-0.29, \mathrm{p}=0.005)$. The SEBT and BMI for age correlated moderately $(\mathrm{r}=0.4, \mathrm{p}<0.001$; $\mathrm{r}=-0.31, \mathrm{p}=0.003$ ) with child-rated quality of life.

\section{Conclusion}

Half of the hypermobile cohort in this study reported experiencing chronic ankle pain which was associated with recurrent episodes of instability. Interestingly, instability and laxity were not correlated in these children suggesting that instability may be neuromuscular in origin. The LLAS may provide more valid quantification of the extent that lower limb joint hypermobility affects physical activity than the more commonly used Beighton score in these children.

\section{Authors' details}

${ }^{1}$ Discipline of Biomedical Science, Sydney Medical School, The University of Sydney, Australia. ${ }^{2}$ Physiotherapy Department, The Children's Hospital at Westmead, Sydney, Australia. ${ }^{3}$ Kids Rehab, The Children's Hospital at Westmead, Sydney, Australia. ${ }^{4}$ Discipline of Paediatrics and Child Health, Sydney Medical School, The University of Sydney, Sydney, Australia. ${ }^{5}$ Endocrinology Department, The Children's Hospital at Westmead, Sydney, Australia. ${ }^{6}$ Discipline of Physiotherapy, Faculty of Health Sciences, The University of Sydney, Sydney, Australia.

Published: 8 April 2014

\section{References}

1. Grahame R: The revised (Brighton 1998) criteria for the diagnosis of benign joint hypermobility. J Rheum 2000, 27(7):1777-1779.

2. Beighton P, DePaepe A, Steinmann B, Tsipouras P, Wenstrup RJ: EhlersDanlos Syndromes: Revised Nosology, Villefranche, 1997. Am J Genetics 1998, 77:31-37.

3. Cohen J: A power primer. Psychol Bull 1992, 112(1):155-9.

\section{doi:10.1186/1757-1146-7-S1-A61}

Cite this article as: Nicholson et al:: Signs and symptoms of foot and ankle dysfunction in children with joint hypermobility. Journal of Foot and Ankle Research 2014 7(Suppl 1):A61.

\footnotetext{
* Correspondence: leslie.nicholson@sydney.edu.au

'Discipline of Biomedical Science, Sydney Medical School, The University of Sydney, Australia

Full list of author information is available at the end of the article
} 